\title{
De Morbis Artificum Diatriba $1700-2000$
}

Juan Manuel Araujo-Alvarez, $\mathrm{M}$ en $\mathrm{C},{ }^{(1,2)}$

José Guadalupe Trujillo-Ferrara, $\mathrm{Dr}$ en $\mathrm{C}$. $^{(3)}$

\section{Araujo-Alvarez JM,Trujillo-Ferrara JG. De morbis artificum diatriba 1700-2000. Salud Publica Mex 2002;44:362-370.}

El texto completo en inglés de este artículo está disponible en: http://www.insp.mx/salud/index.html

\section{Resumen}

Bernardino Ramazzini, considerado el padre de la medicina del trabajo por haber escrito el primer tratado sobre las enfermedades de los trabajadores (D e MorbisArtificum Diatriba) nació en Capri en 1633, fue médico de renombre y escritor prolífico. El Tratado sobre las enfermedades de los trabajadores contiene el análisis de 53 profesiones, un método particular y específico de análisis y propone, del mismo modo, una metodología para evitar la ocurrencia de estas enfermedades. En este ensayo se reivindica el modo de proceder del padre de la medicina del trabajo, se acepta la vigencia de los principios planteados en el Tratado y se hace justo homenaje al hombre y su obra en el tercer centenario de su publicación. El texto completo en inglés de este artículo está disponible en: http://www.insp.mx/salud/ index.html

Palabras clave: enfermedades de los trabajadores; metodología de análisis; medidas preventivas; homenaje

\author{
Araujo-Alvarez JM,Trujillo-Ferrara JG. \\ De morbis artificum diatriba 1700-2000. \\ Salud Publica Mex 2002;44:362-370. \\ The English version of this pape \\ is available at: http://www.insp.mx/salud/index.html
}

\section{A bstract}

Bernardino Ramazzini was a renowned physician and a prolific writer, born in Capri in 1633. He is considered the father of occupational medicine for having written the first paper on workers' diseases (De morbis artificum diatriba). H is Treaty on W orkers' D iseases included 53 different professions, one particular and specific method of analysis, and a methodological proposal to prevent these diseases. This essay supports the approach taken by the father of occupational medicine and confirms that the principles established in his work are applicable to this day.A fair tribute is paid to the man and his writings in the third century after their publication. The English version of this paper is available at: http://www.insp.mx/salud/index.html

Key words: diseases of workers; methodology of analysis ; prevent measures; homage
$\mathrm{H}$ ace 300 años, en 1700, apareció en Italia una obra considerada desde entonces por muchos como la piedra angular de la medicina del trabajo. Se trata de la obra de Bernardino Ramazzini De Morbis Artificum Diatriba (Tratado sobre Las enfermedades de los tra- bajadores). Por esta razón, al magister de Capri se le considera como el padre de la medicina del trabajo. Por las cartas dirigidas por Ramazzini a Antonio Magliabecchi, bibliotecario del Gran Duque de Toscana, desde el 24 de mayo de 1692, en las que va rela-

(1) Departamento de Salud Pública y Ciencias Sociales. Escuela Superior de Medicina, Instituto Politécnico N acional (IPN). México, D.F., México.

(2) Departamento de Atención a la Salud. Universidad Autónoma Metropolitana Unidad Xochimilco. México, D.F., México.

(3) Departamento de Bioquímica. Escuela Superior de Medicina, IPN . México, D.F., México.

Fecha de recibido: 28 de marzo de 2001 - Fecha de aprobado: 15 de mayo de 2002

Solicitud de sobretiros: M en C. Juan Manuel A raujo Alvarez. Departamento de Salud Pública y Ciencias Sociales. Escuela Superior de Medicina, Instituto Politécnico N acional. Plan de San Luis y Díaz Mirón, Casco de Santo Tomás, México, D.F. Correo electrónico: jmaraujo@ latinmail.com 
tando las necesidades y dificultades para escribir la obra por la cual será más famoso, se conocen los antecedentes sobre la creación de la misma. El 22 de octubre de 1700 comunica a Magliabecchi: "He terminado de escribir mi opúsculo Las enfermedades de los trabajadores (...) En siete u ocho días debo partir a Padua para su lectura". ${ }^{1}$

Bernardino Ramazzini nació en Capri, en una familia acomodada, el 4 de octubre de 1633. Estudió latín y griego. A los 26 años obtiene el reconocimiento doctoral de medicina y filosofía. Desde 1663 trabaja como médico municipal en Canino y Marta, dos pequeñas ciudades del ducado de Castro. Desde entonces, participa y lo invitan a participar en numerosas academias y se relaciona con los intelectuales más renombrados de su época. Profesor en Modena desde 1682. La academia Imperial Leopoldo-Carolina de Naturae Curiosorum de Viena le otorga un diploma en el cual viene señalado "tercer Hipócrates".

Desde 1690, y hasta 1694, Ramazzini compone las Constituciones epidémicas, una aportación fundamental, como las elaboradas por Hipócrates. En estas publicaciones son tratadas las relaciones anuales sobre las enfermedades que se presentaban en el territorio modenese. Otra afirmación que se plantea en estos escritos es sobre la necesidad (en el sentido de la mayor eficacia) de prevenir las enfermedades, desde el momento en que las curas utilizadas para controlarlas no aportan resultado alguno. De igual modo tiene importancia el planteamiento que se hace sobre la medicina pública, de la iniciativa permanente del Estado y no solamente en ocasión de las terribles epidemias, de interesarse por la salud de la población que representa. Estas constituciones de Ramazzini sirvieron de ejemplo para que se realizaran otras cincuenta en diversos países de Europa.

Desde el año académico 1690/1691 lleva a cabo su curso de medicina acerca del Estudio modenese, dándole como título De Morbis Artificum. Seguramente estos fueron los prolegómenos de su obra maestra, pues en relación con ésta, los indicios que se conocen comienzan con las cartas dirigidas a Antonio Magliabecchi, por lo que se puede pensar que el tratado fue escrito a lo largo de 10 años y terminado cuando el autor tenía 67 años de edad y unos 40 de práctica médica. La edición de 1700 publicada por los tipógrafos de A. Capponi contenía 40 capítulos* y una disertación so-

\footnotetext{
* Por un error tipográfico se omitió el capítulo 8, entre el 7 y el 9, por lo que aparecen en la numeración final 41 capítulos. Esta omisión se ha venido conservando, tanto en las siguientes ediciones como en todas las traducciones posteriores.
}

bre las enfermedades de los intelectuales indicada como "último capítulo". La edición de 1713 es la última preparada por el autor y comprende el Tratado sobre las enfermedades de los trabajadores, publicado por primera vez, un suplemento de doce capítulos dedicados a otros tantos grupos de trabajadores, escrito a propósito para la edición paduana, aunque es omitido el capítulo sobre las enfermedades de los albañiles, y una disertación sobre las enfermedades de las monjas.

Esta novedosa visión de investigador y clínico, no obstante la situación social, política, económica, así como las dos visiones contrapuestas del universo que prevalecían en su época (el mecanicismo y el panvitalismo) le permite identificar los cambios introducidos, en la existencia individual y social, por los procesos productivos denominados "modernos" que se traducen en un modo de vivir que da lugar a nuevas enfermedades, así como identificar los mecanismos responsables y proponer una terapéutica, aunque muy limitada, específica; pero lo más importante, recomendaciones que involucran ámbitos diferentes, para evitar la aparición de estas enfermedades.

\section{Desarrollo del tema}

A Bernardino Ramazzini le tocó vivir una época de crisis económica estructural que se refleja en grandes revoluciones espirituales en Europa: el llamado mundo barroco. Tiempos de gran polarización social entre burguesía, clase media y una cada vez mayor masa de pobres que despertaba, hacia los demás, reacciones ora de rechazo, ora de atención. Al mismo tiempo una crisis de los Estados tradicionales, guerras prolongadas y desestabilización moral.

Las soluciones a la crisis iban desde la intervención del Estado en la articulación de una economía en desorden, hasta reivindicar a la monarquía absoluta como fuerza rectora e impositiva para restaurar el orden social, el desarrollo de la ciencia racionalista nueva como medio para acabar con la dispersión de las ideas que no podían ser organizadas por el saber griego y medieval; hasta la reorganización geopolítica de Europa así como la disminución del poder papal como medida para acabar con los grandes imperios económicos. ${ }^{2}$

Sólo hasta finales del siglo XVII empezó una lenta recuperación, luego de la revolución inglesa de 1688. Pero, si bien todo esto vale para Europa, mientras tanto Italia, a decir de Gramsci, ${ }^{3}$ después del año 1000 inicia la reacción contra el régimen feudal; en los dos o tres siglos siguientes se transforma profundamente el ordenamiento económico, político y cultural de la so- 
ciedad; se revigoriza la agricultura, se reavivan, extienden y organizan las industrias y los comercios; surge la burguesía, nueva clase dirigente, con ardiente pasión política y unida en poderosas corporaciones europeas, se reconstituye con creciente espíritu el Estado comunal. No obstante, el "Estado" en el estado comunal tuvo un significado "corporativo" limitado pues no pudo desarrollarse más allá del feudalismo medio. En Italia los Comunes no supieron salir de la fase corporativa, la anarquía feudal predominó y luego vino la dominación extranjera.

Pero en este proceso de alternativas y contralternativas socioeconómicas y políticas para superar la crisis es la ciencia la que recibe uno de los más grandes impulsos de su historia, con base en el enfrentamiento entre lo viejo y lo nuevo, en aras de perfilar un paradigma epistemológico capaz de lograr acuerdos entre los científicos para desarrollar una ciencia normal. ${ }^{4}$ Pues como dice Koyré, al estudiar la historia del pensamiento científico y filosófico de los siglos XVI y XVII “... tan íntimamente interrelacionados entre sí que, si se separan, se tornan incomprensibles, me he visto forzado a reconocer (...) que durante este periodo el pensamiento humano, o al menos el europeo, sufrió una profunda revolución que transformó el marco y los patrones de nuestro pensamiento, de que la ciencia y filosofía modernas constituyen a la vez la raíz y el fruto". 5

El núcleo del nuevo paradigma fue poner en claro la función de los sentidos y de la razón, es decir de sus interrelaciones, en el proceso del conocimiento científico, una vez que el concepto mismo de conocimiento científico hubiese sido definido. Pero ante la imposibilidad de lograr establecer qué era y qué caracterizaba al conocimiento científico, el problema se trasladó a los intentos de resolver las preguntas de ¿cuál es la función de los sentidos y cuál la de la razón o cuáles son sus interrelaciones en el proceso de conocimiento científico? Para unos la respuesta era que el conocimiento verdadero se adquiriría por los sentidos y por un proceso de inducción; para otros el conocimiento es verdadero a través de la relación de ideas racionales y ello sólo era realmente válido cuando se lograba conocer cómo funcionaba la razón misma que conoce; para otros el conocimiento objetivo es fruto de la síntesis entre empirismo y racionalismo o entre inducción y deducción. ${ }^{2}$

Para el estudio de la enfermedad, en el barroco, el empirismo y el racionalismo fueron las bases filosóficas, que se expresaron en la división de una medicina práctica y otra teórica. La primera, fue la de los clínicos propiamente dichos, y siguió sobre todo el camino de los empiristas. La segunda, siguió el camino matemático aplicado a la experimentación y a través del trabajo de los iatromecánicos y algunos iatroquímicos.

En el camino de los empiristas destacarán tres corrientes: el empirismo clínico de Sydenham, el empirismo etiológico de Van Helmont y el empirismo anatomopatológico de Boerhaave y Albertini. ${ }^{2}$

En este contexto se inscribe el esfuerzo de Ramazzini y su De Morbis Artificum Diatriba.

Pero, para Ramazzini, la elaboración de esta obra es un compromiso desde el momento en que él ejerce precisamente la medicina práctica, pareciéndole de poca relevancia tratar aspectos teóricos o abstractos que no tienen ninguna aplicación, pues considera es un tema de enorme interés para la sociedad, en la medida en que el trabajo y quienes lo realizan, trátese de trabajo intelectual o manual, merecen ser protegidos, promovidos, y ofrecérseles incentivos y privilegios pues gracias a los trabajadores, el Estado y las ciudades cuentan con riquezas. ${ }^{5}$

La idea de escribir un tratado sobre las enfermedades de los trabajadores, a decir de Ramazzini, surge cuando observa cuán penosa es la labor de un trabajador dedicado al vaciamiento y limpieza de las cloacas. ${ }^{6}$ Actividad que se ve obligado a realizar este trabajador y de la cual piensa obtener el alimento para prolongar su vida y la de su propia familia, pero de ésta le derivarán molestias y enfermedades muy graves e incluso la muerte. Por esta razón, los trabajadores maldicen el trabajo en el que habían puesto su esperanza de vida. ${ }^{5}$

Los aspectos clínico y sanitario del Tratado sobre las enfermedades de los trabajadores son de gran interés. Se trata del primer tratado sistemático de patología del trabajo y que hasta algunos siglos y decenios antes no podía ser definido ni completo, ni mucho menos actual. ${ }^{7}$ Ramazzini, más que anticiparse al tiempo, atestigua el momento en el que se comienza a delinear un cuadro de patologías que, con el paso de los años, se han propagado más que las demás y se han vuelto masivas para las clases subalternas, debido al nuevo orden social, político y económico que se va gestando y que se convierte en una segunda naturaleza para el hombre.

La novedad es haber descrito por separado la cuota de patología infecciosa, es decir la de las epidemias, de las que tienen como principal característica un aspecto evolutivo, es decir, las crónicas. En este tipo de observaciones el clínico Ramazzini es efectivamente un innovador. Muy cierto es que, en general, tal vez no hizo otra cosa que sistematizar y dar orden a una 
gran cantidad de datos y observaciones que ya existían, ${ }^{8}$ pero el buen resultado de esta síntesis estuvo asegurada, además de las motivaciones que lo inspiraron, por el método adoptado, producto siempre de la verificación directa de las observaciones existentes y de la crítica a las interpretaciones dogmáticas e irracionales de su época. Prueba de ello fue su exigencia fundamental de corroborar en los cadáveres la existencia de aquellas alteraciones que su intuición clínica ya había reconocido en los órganos internos de los pacientes para remontarse a los hechos constatados por las leyes de la patología. ${ }^{8}$ Un conocimiento que empezó a erigirse como una rama médica de capital importancia, tanto para la clínica como para la enseñanza y la investigación que, en Padua, desde mediados del siglo XVI se institucionalizó como la investigación de las lesiones en el cadáver ${ }^{2}$ y que con Lancisi, de ser un mero hallazgo, pasó a ser clave del diagnóstico, ${ }^{2}$ al igual que para Morgagni para quien ésta se debería llevar a cabo, en los enfermos que morían, para confirmar gran parte de la hipótesis diagnóstica del médico, lo que también era formulado por Ramazzini, ${ }^{9}$ en esta obra clásica.

Inicia diciendo: "Las causas que provocan, desde mi punto de vista, las diversas y graves enfermedades de los trabajadores son dos (...) La primera causa, la más importante, está representada por las propiedades de las sustancias usadas que, produciendo gases y polvos tóxicos, inducen enfermedades particulares; la segunda está representada por aquellos movimientos y por aquellas posturas no naturales por las cuales la estructura misma del cuerpo resulta dañada, de tal forma que con el tiempo aparecen de improviso enfermedades graves ...". ${ }^{5}$

A lo largo de los 29 primeros capítulos habla sobre "... los trabajadores que se enferman a causa de las substancias usadas ...". ${ }^{5} \mathrm{~A}$ partir del capítulo 30 dice "... tomaremos bajo examen a aquellos que enferman por otras causas como las posiciones y los movimientos no fisiológicos del cuerpo ...".

Incluye además a los atletas, a los cantantes, a los soldados, a los intelectuales, a los marineros y a las monjas. Es decir, a toda una gama de actividades sociales que hoy día, por alguna extraña razón, han quedado fuera del ámbito de la atención de los médicos dedicados a atender los problemas de salud de los trabajadores; aun cuando su contrasentido es que precisamente hoy estas actividades obligadamente deben ser consideradas como laborales.

El método usado por Ramazzini en el tratado, en cada capítulo, puede ser esquematizado de la manera siguiente:
1. Descripción de la tecnología

2. Examen clínico del trabajador, dirigido a verificar los efectos probables derivados del trabajo desarrollado, además también dirigido a verificar los mismos efectos ya conocidos en otros trabajadores que han desarrollado la misma profesión, arte u oficio y que, más en general, pertenecen al mismo estrato social

3. Revisión de la literatura, de la experiencia ya existente sobre el tema

4. Discusión de la terapia, de los remedios que se aplican tanto a los individuos como también al ambiente de trabajo

5. Propuesta de norma de comportamiento, de vida, de trabajo, de carácter más general y por tanto de obligación para sustituir una norma peligrosa pero impuesta por las costumbres, por ordenamiento social y más en particular por la injusticia, por la codicia de la ganancia producto de este orden social". ${ }^{6}$

Veamos un breve ejemplo:

\section{Capítulo cuarto}

\section{Las enfermedades de los químicos}

Los químicos se jactan de conocer la técnica de volver inocuos a todos los minerales, sin todavía evitarse en ellos mismos su toxicidad; en efecto la mayoría se enferman de las mismas enfermedades de aquellos que trabajan los minerales $y$, si en charlas lo niegan, lo revelan con el color del rostro. Leonardo de Capua refiere que Teofrasto y van Helmont, dos químicos célebres, se enfermaron gravemente preparando medicamentos. Juncken, en su Chimica sperimentale, dice que inhalando los polvos de antimonio durante la fabricación del vidrio de antimonio, los trabajadores se tornaron asmáticos y fueron atacados por vértigos. Ettmüller dice que, en la preparación de la lavativa de antimonio, habiéndose quebrado por casualidad la retorta, "absorbí el vapor del azufre y del antimonio" y él, que estaba completamente sano, enfermó de una tos que le duró cuatro semanas; atribuyó la causa de esta tos exclusivamente al humo ácido que le había irritado los órganos de la respiración. Es muy curioso que Tachenio, en su Ippocrate chimico (Hipócrates químico), dice que por casualidad pretendía sublimar el arsénico para fijarlo; "después de muchas operaciones abrí el envase y advertí con sorpresa un olor agradable; pasada una media hora tenía dolores en el estómago, como de úlcera, con dificultad para respirar, sangre en la orina, cólicos 
y temblores en los miembros". El autor dice luego haberse repuesto un poco tomando aceite y leche, pero que durante todo el invierno fue atormentado por una fiebre continua similar a la fiebre de los tísicos, de la cual se liberó con un cocimiento de hierbas suaves y comiendo las puntas de col. "Yo conocí a Carlo Lancillotto, químico por nuestra región muy renombrado, que estaba afectado por temblores y por una enfermedad de los ojos, estaba sin dientes y asmático, maloliente y con tal aspecto disminuyó la fama y el prestigio de sus medicamentos, particularmente la de los cosméticos de los cuales alababan su eficacia". Sin juzgar cierta fatiga todavía derrochaba un empeño de este género; así son admirables los químicos que se dedican con seriedad al estudio de sustancias poco conocidas y se arriesgan en las ciencias naturales, sin temer sacrificar la propia vida por el bien de todos. Ni se les debe culpar por el hecho de que no consigan protegerse suficientemente de los efectos venenosos de aquellas sustancias que buscan transformar en medicina. Es necesario que estén presentes y observen todas las fases de los procedimientos cerca de la llama del fuego y del humo del carbón, es indispensable que los medicamentos sean preparados según las reglas y puedan ser administrados sin riesgo. En efecto la mínima variación y negligencia, en manipular los productos químicos, puede modificar a tal punto la calidad para hacerlos pasar, como dice Renato Cartesio, a la categoría de los venenos. A esta precaución Juncken, en su prefacio, afirma que los fármacos preparados químicamente no pueden ser administrados por el médico con tranquilidad si no habían sido elaborados por él mismo o por un químico experto, bajo sus propios ojos. Por lo consiguiente no se debe culpar a un picador si al domar a un caballo fiero y rebelde viene a veces decepcionado y encogido de los pies, así no se debe decir del químico que sale de su laboratorio lívido y asustadizo como un ser proveniente de ultratumba.

Algunos años atrás, surgió una importante disputa entre un ciudadano y un comerciante modenese pues, en un país de esta jurisdicción llamado Final, existía un gran laboratorio para la producción del sublimado. El finalese hizo comparecer en juicio a este comerciante, exigiendo transferir su laboratorio fuera del país o en otro lugar, desde el momento en que envenenaba todo el vecindario cuando en las obras, para obtener el sublimado, quemaban el vitriolo. Para probar la exactitud de su acusación exhibía el testimonio del médico de aquel país y por otro lado el registro parroquial de muertes. Por este último resultaba evidente que en aquel país y en la zona más próxima al laboratorio morían cada año más ciudadanos que en otros países. El médico por otro lado podía atestiguar que los ciudadanos que vivían alrededor del laboratorio morían principalmente de consunción y de enfermedades pulmonares, atribuidas sobre todo al vitriolo que se difundía y contaminaba el aire circulante volviéndolo dañino para los pulmones. El doctor Bernardino Corrado, comisario de artillería en el ducado estense, asumió la defensa del comerciante, mientras que el doctor Casina Stabe, por entonces médico de aquel país, defendió al ciudadano de Final. Por una parte y por la otra se produjeron muchos documentos interesantes en los cuales se discutía finamente sobre los posibles efectos de la nube de vitriolo. Al final los jueces dieron la razón al comerciante y el vitriolo fue declarado inocente y absuelto. Si el experto del derecho en este caso hubiera juzgado bien, debió legar el juicio a los expertos de las ciencias naturales.

Para volver a mi argumento, me abstendré de ofender a los químicos al proponer cualquier remedio, ya sea preventivo o curativo, para todas las situaciones en las cuales del oficio se obtiene más daño que ganancias. En efecto, no conozco ninguna enfermedad por sanar para la cual los químicos no tengan remedio precioso y listo en el armario, por eso prefiero ir delante y examinar otras profesiones. ${ }^{5}$

Como se puede observar, la tecnología o más simplemente el modo de trabajar y los materiales implicados en el taller artesanal y en la agricultura representaron los intereses precisos para el estudio de Ramazzini, que se expresa en cada uno de los capítulos de esta obra, lo mismo que en la descripción de los ambientes de trabajo. Y es que para el autor si bien el conocimiento de las condiciones de trabajo y, sustancialmente también de los materiales de trabajo observados y descritos por él, tienen como finalidad estudiar los efectos que sobre el hombre ejercen, también este interés estaba motivado, por una parte por la esperanza de superarlos y así aumentar la productividad con un medio diferente al de la intensificación del trabajo y, por otra, para contribuir a obtener el éxito, más allá del nivel individual, desde una perspectiva social, de los valores humanitarios y científicos que condujeran como resultado a la conservación de la fuerza de trabajo.

Por lo tanto, es mérito innegable de Ramazzini haber dicho que no es degradante para los médicos visitar y frecuentar sistemáticamente, para comprender mejor y curar algunas enfermedades, los ambientes de trabajo, los talleres de los artesanos.

Para Ramazzini, visitar el lugar de trabajo era la lógica y coherente continuación del proceso que inicia con la pregunta que hace el médico al paciente, sobre el tipo de trabajo desarrollado. Pero en aquel mo- 
mento histórico, la novedad verdadera y propia estaba, más que a propósito de la pregunta sobre el trabajo desarrollado y la visita al lugar de trabajo, precisamente por la ocasión de intervenir o investigar, para curar, un enfermo que fuera trabajador. Ramazzini es sustancialmente un activo y atento testigo de tal encuentro entre médico y trabajador que obviamente no es casual ni producto de su simple iniciativa, sino de las condiciones materiales de vida de la época. Por tales razones destaca opiniones y hace constantes referencias a quienes han dejado claro las causas de tales condiciones de vida y, en consecuencia, de trabajo; como cuando cita a Ovidio, quien refiriéndose a los males que corrompen a las almas y las buenas costumbres, critica la codicia y locura de los hombres que obligan a otros a penetrar en las entrañas de la tierra, y en este mismo sentido señala cómo por estas mismas razones la mortalidad de los que extraen los minerales de las minas es habitualmente muy elevada, que sus mujeres se han casado hasta siete veces. ${ }^{5} \mathrm{O}$ cómo destina un capítulo para analizar las enfermedades de los hebreos de quienes dice esta "nación (...) no tiene comparación y no se parece a ninguna otra (...) en cuanto a que no tiene sede por ninguna parte, sin embargo está por doquier, es ociosa y trabajadora al mismo tiempo, no ara, no trilla, no siembra, ni cosecha; (...) por consiguiente, no por motivos de raza, como se cree comúnmente, y ni siquiera por sus costumbres particulares, sino por el tipo de trabajos desarrollados es afligida por diversas enfermedades $(. ..){ }_{1}^{5}$ para sólo mencionar dos ejemplos de los muchos que aparecen en esta obra.

Del mismo modo, el clínico Ramazzini expresa otra característica fundamental en el Tratado sobre las enfermedades de los trabajadores, se trata de la síntesis entre los problemas sanitarios y los de tipo técnico, respecto al fin que está propuesto: esto es, evitar o por lo menos reducir una serie de enfermedades a quienes trabajan. Su competencia directa sobre muchos campos del conocimiento, por una parte, y la relativa simplicidad de los aspectos técnicos a profundizar, por la otra, le facilitaron el logro de una síntesis suficiente. Destaca en su razonamiento la marcada subordinación de las observaciones técnicas respecto a las clínico-sanitarias y más en general la no rigidez de esta última. Y es que, muchas veces le ocurre al mismo Ramazzini, encontrándose de frente a un trabajador enfermo, que debe abandonar el esquema diagnóstico e incluso el curativo adoptado habitualmente en la confrontación de las enfermedades. Por lo que en un proceso de este género sustancialmente son sometidas a discusión toda la lógica y la cultura prevalentes, sin por esto caer en otro exceso: querer encontrar a cualquier costo las razones de una enfermedad en los materiales y en las condiciones de trabajo. Esta forma de proceder que para Ramazzini parece un razonamiento confiable al mismo tiempo que de máxima libertad, le permite el uso de todos los instrumentos interpretativos de los cuales podía disponer para una investigación sin obstáculos.

Ramazzini redimensiona sistemáticamente la eficacia de la terapia sobre múltiples formas morbosas y, en particular, sobre algunas que atacan más frecuentemente a los trabajadores, pero principalmente creía verdaderamente en la posibilidad y en el beneficio de evitar tantas enfermedades, por eso señala como una atrocidad de la sociedad hipócrita de la época la clausura de las termas que le permiten a los trabajadores asearse y obtener con esto un descanso de la ardua labor; o repudiar insistentemente el uso de las sangrías pues, debilitan aún más el cuerpo de los de por sí extenuados trabajadores; o como Hipócrates, deposita su confianza en la alimentación requerida, ya únicamente para recuperar las fuerzas perdidas en el trabajo o indispensable en cierto tipo de profesiones. Este último aspecto ha sido pobremente indagado por cuantos han escrito sobre la obra de Ramazzini. En efecto, las indicaciones preventivas, las indicaciones consideradas como medidas técnicas a adoptar para evitar la exposición a polvos, humos, etcétera, ocupan pocas líneas en algunos capítulos en los cuales es muy extensa la parte terapéutica, muy repetitiva frente a las causas muy variadas de las enfermedades. Se trata en cambio de indicaciones muy precisas que por otro lado son comprensivas por el juicio de aplicabilidad y de eficacia.

Por otro lado, Ramazzini no insiste sobre indicaciones obvias o radicales, pero repite el concepto según el cual el bienestar de los trabajadores depende de la confluencia de diversas situaciones, algunas internas al puesto de trabajo, múltiples y engranadas entre sí, y otras, igualmente determinantes, externas al lugar de trabajo, de carácter social. Por cuanto interesa al primero, lo destaca generalmente, en especial cuando comprueba en varias ocasiones su hipótesis de fondo, sobre la causa de las enfermedades del trabajo. Examina separadamente por una parte la substancia elaborada, casi siempre relacionada desde el punto de vista de los efectos sobre la salud de quien ha estado en contacto, y por el otro, los movimientos, el orden y por eso la organización de los movimientos adoptados para modificar y elaborar aquella sustancia. Se trata de señales precisas, en el sentido causal, de la organización del trabajo y las relaciones económicas que someten al trabajo y que derivan mecánicamente. 
Es una temática muy importante para ser reexaminada. Es interesante notar que Ramazzini la intuye y le da toda la importancia que tiene en realidad también a propósito de la salud de quienes trabajan, el médico, que debería por tanto intentar curar las enfermedades, no todas, exclusivamente con los fármacos contenidos en la farmacopea de su tiempo. En este sentido el autor plantea los problemas y revela las contradicciones que nos parecen por lo menos ingenuas, pero que tenían el valor, en aquellos años, de ser correctas, justas y que como tales habían sido desatendidas, mistificadas activamente por quienes eran responsables de la respuesta a estas cuestiones y por quienes habían podido mantener esas contradicciones. Las contradicciones planteadas por Ramazzini se encuentran sobre un abanico muy amplio por importancia y por urgencia. Para él los problemas derivan del hecho de que la sociedad debería proteger a los mismos trabajadores, del hecho de que el trabajo, necesidad material y también obligación moral, se apropia de la libertad para corresponder con esclavitud y fatiga, forja la civilización, pero al mismo tiempo destruye la vida humana.

Por tanto, el punto central de la obra de Ramazzini es el siguiente: el obrero enfermo, sufre y muere muy frecuentemente en su trabajo, actividad que debe realizar para vivir tanto él como su familia, y también valiosa para la sociedad, porque sin trabajo no es posible el progreso de ésta; por lo tanto el trabajador debe ser protegido, mantenido y preservado. ${ }^{10}$ Por otra parte, estaba profundamente convencido de la importancia de las artes mecánicas para el progreso de la civilización. Lleva adelante esta convicción, además de mantener las creencias de la medicina clásica que ha aprendido en los años de estudio y aplicado en su práctica clínica y que se inspiran todas, fundamentalmente, en los preceptos de la naturaleza: "en la naturaleza la enfermedad, en la naturaleza el remedio", como enseña Hipócrates y repiten Sydenham y Baglivi. ${ }^{6}$

Desde el momento en que afirma que el médico, cuando se dispone a examinar a un enfermo, debe interrogarlo amén de sus trastornos y sobre su alimentación, también sobre la ocupación a la cual se dedica, cumple simultáneamente un acto de fe y una profunda revisión respecto a las enseñanzas hipocráticas. La medicina hipocrática que se funda sobre el equilibrio entre el organismo vivo y de su ambiente, considera la enfermedad como un esfuerzo por reconstruir el equilibrio alterado y la intervención del médico en un acto de cooperación con la naturaleza en sus esfuerzos por el logro de la cura.
Es por ello que cuando se define a Ramazzini, como muchos lo han hecho, como hombre renacentista o preiluminista, ${ }^{11,12}$ esta definición tiene sustancialmente el mismo significado que cuando se quiere sostener que, con su obra y sus escritos, se proponía contribuir al logro de niveles de vida más humanos, tendientes a la "felicidad" o a la tranquilidad de la mayoría de los elementos que componen a la sociedad.

Resulta pues de gran interés, por una parte, la síntesis de todos los conocimientos médicos trabajados por el autor y, por el otro, la operación efectuada en el sentido de organizar la síntesis en función de un estrato social preciso, los trabajadores, en beneficio de éstos, incluso, en perspectiva de toda la sociedad. Ramazzini conoce y estudia, casi siempre directamente, los materiales y las formas de trabajar de su tiempo e intenta conectar en un informe muy preciso a éstos con la morbilidad y la mortalidad de los trabajadores. Cierto es que hoy podríamos afirmar con más seguridad que no todos estos modelos causales correspondían a la realidad o que correspondían sólo en parte. En este sentido no sería más que suficiente una simple actualización de la obra de Ramazzini para ilustrar las actuales condiciones de trabajo y de salud de los trabajadores. En el horizonte de tres siglos han surgido y ordenado al menos dos hechos que atañen a la patología: se han reducido las enfermedades infecciosas como causa de muerte; los agentes químicos o físicos de las enfermedades han operado, en condiciones diversas en este horizonte de tiempo, pero de manera tal que expresan más directa y suficientemente las enfermedades y por eso también la causa de muerte; al mismo tiempo que se han operado profundos cambios en el orden social, económico y político que han dejado cada vez más claro que son éstas las verdaderas condiciones que determinan la salud no sólo de los trabajadores, sino también de la sociedad en su conjunto, como ya vislumbra Ramazzini. Vale decir que mientras los artesanos de Ramazzini morían casi todos prematuramente por las mismas enfermedades o por una variedad muy reducida de enfermedades, es decir principalmente por complicaciones infecciosas, con el paso del tiempo las enfermedades del trabajo como tales, por los materiales y por las condiciones de trabajo, sin ser eliminados, y muy por el contrario se han incrementado a la vez que diversificado, se convirtieron en una de las causas principales de la morbilidad y de la mortalidad.

Estos serán los principios de toda una tendencia en la interpretación de los fenómenos relacionados con la salud de los trabajadores, después vendrán Johann Peter Franck (...) y Rudolph Virchow (...), incluyendo 
a Friedrich Engels (...), (...), lo que ha permitido rebasar la concepción tradicional, aunque todavía hegemónica, de que los accidentes, la invalidez y las enfermedades son producidas por el trabajo como un factor de riesgo ambiental, pues obliga al trabajador a estar en contacto con agentes químicos, físicos, biológicos y psicosociales; y por lo tanto considerar que los riesgos ocupacionales son producto de la organización social a que son sometidos los trabajadores. ${ }^{13}$

Por eso, dice el doctor Jesús Kumate: "sorprende que los determinantes laborales registrados hace tres siglos, con los matices del progreso, sean los mismos, por ejemplo: a las enfermedades por polvo (...) se han agregado (...) el asbesto, el ántrax de los cardadores, miles de compuestos químicos orgánicos e inorgánicos, la mayoría tóxicos en grado variable; (...). Al ruido (...) la hipoacusia de los músicos de rock a edades muy tempranas o la experiencia desagradable de las jornadas de la bolsa y los empleos de pista en aeropuertos. Al ambiente físico (...) se agregan ahora las radiaciones ionizantes de los rayos $X$, la industria nuclear, los vuelos estratosféricos y los espaciales $(\ldots)^{\prime \prime},{ }^{\prime 14}$ para sólo mencionar algunos.

Para hacer el diagnóstico de enfermedad de trabajo Ramazzini no se limita a considerar la disposición de los elementos clínicos, debe superar las dificultades equiparables a aquellas que encuentra un médico de hoy de frente a una enfermedad crónica o de frente a los indicadores precoces de enfermedad. Debe por eso estudiar el ambiente de trabajo y adoptar modelos interpretativos particulares. Encargarse, como pueda, de aquellas enfermedades, y se comprende que la exigencia por una terapia más adecuada, enfocada en la enfermedad del trabajador de su época no corresponde a otra cosa sino a la exigencia, ahora no completamente expresada, de hacer entender que la mayoría de las enfermedades no sólo son curables sino que además son prevenibles.

\section{Conclusiones}

Ramazzini afirma, como decía Sydenham, que la enfermedad no se conoce por los libros ni por las ciencias fundamentales, sino observando directamente al enfermo en su lecho, pero va más allá, pues observa además las condiciones de vida de tal enfermo; tratándose de un trabajador, le interesa intentar encontrar las causas de la enfermedad en cuanto "esencias". Por eso se le considera, como a Fracactoro y a Paracelso, en la historia de la medicina, como uno de los iniciadores del pensamiento etiopatogénico. ${ }^{2}$ Quiso, como Hipócrates y Sydenham, conocer las enfermedades en cuanto especies generales y no sólo a enfermos particulares, por eso procede como botánico y como los pintores manieristas clásicos, para establecer especies clínicas; es un baconiano convencido de que lo apropiado en la ciencia es "descubrir" y no "inventar o imaginar" la naturaleza, aunque también la imagina y reinventa. Es consecuente con lo que plantea Sydenham sobre que la labor del médico ha de ser "descubrir" cada enfermedad por sus características específicas que la hacen única y claramente distinta de las demás.

Ramazzini también actúa, según Van Helmont, considerando que una enfermedad es generada por una semilla específica productora, con base en un plan determinado. Pero va más allá pues también considera que es resultado de un desequilibrio de los humores o de la descomposición de la materia orgánica.

Ramazzini también es un empirista anatomoclínico como Boerhaave y Albertini, pues trató de identificar objetivamente cada enfermedad de la que hablaba y, como Lancisi, efectuaba o sugería la realización de autopsias para tratar de correlacionar los hallazgos anatomopatológicos con los antecedentes clínicos.

Ramazzini, como Boerhaave, ${ }^{2}$ continuando la obra de Hipócrates, restructuró la historia clínica con el capítulo de antecedentes laborales e historia laboral, al agregar la simple pregunta ¿en qué trabaja usted? Además de una actitud ante el enfermo-trabajador, comprensiva e interesada, tanto de visitar el lugar de trabajo como el sitio donde vive, lo que no debe apenar al médico.

Ramazzini también contribuyó al estudio de la enfermedad como fenómeno colectivo o epidemiológico. Fue lo que Entralgo, ${ }^{15}$ llama un "sabio jánico" hombre eminente cuyo saber tenía dos rostros, uno claramente orientado hacia su presente y futuro, y otro -aunque no por espíritu reaccionario o por pura nostalgia- vuelto todavía hacia el pasado, hacia el magisterio de la sabiduría antigua. Estas fueron las virtudes y limitaciones de Bernardino Ramazzini.

El extraordinario esfuerzo de Ramazzini nos debe servir de ejemplo para enriquecer nuestro saber médico con una concepción política de salud que nos conduzca a precisar conceptualmente el proceso que escindió lo político, social e ideológico del problema salud-enfermedad y que se formalizó en lo que se denominó el modelo médico, cuyas características (biologismo, individualismo, ahistoricidad, asocialidad, mercantilismo y eficacia pragmática), han impedido la comprensión de que la salud de los trabajadores (...), es el resultado de dos momentos de un mismo proceso, el proceso de trabajo, que se realiza bajo determinadas leyes y normas, que corresponden 
a un modo de producción determinado, (...) el capitalista. ${ }^{16}$

Se puede afirmar entonces que la salud de los trabajadores en el capitalismo es, en términos concretos, el resultado de las relaciones que se dan en el marco de la explotación de la clase trabajadora y de la sociedad en su conjunto. Esto es lo que caracteriza al capitalismo en cualquiera de sus etapas históricas, formas o disfraces que adopte, incluyendo el neoliberalismo actual. ${ }^{17}$ Por lo tanto, queda claro que, en tanto no superemos esta forma de organización social por una más humana, plural, inclusiva y democrática no resolveremos los problemas de salud de los trabajadores que enfrentamos hoy día, pero, en tanto esto sucede, contamos con una amplia experiencia histórica y un bagaje técnico y científico suficiente para dar pasos importantes en la resolución de esta problemática que, incluso, puede rebasar el ámbito laboral.

Importante es pues, para todos los médicos, conocer esta obra clásica y revalorar este campo de estudio que hoy día cobra cada vez más vigencia, tanto en nuestro país como en el resto del mundo. Nosotros, por medio de estas notas, queremos rendir un justo homenaje a Bernardino Ramazzini, padre de la medicina del trabajo, en el tercer centenario de la publicación de su obra El Tratado sobre las enfermedades de los trabajadores, publicada en 1700 y reditada en 1713.

\section{Referencias}

1. Ramazzini B. Carta a Magliabecchi, del 20 de junio 1700. En: Epistolario, publicado en ocasión del CCL aniversario de su muerte a cargo de Pericle Di Pietro. Modena, Stab. Tip. P. Toschi \& C. 1964, pp. 232-233. En: Carnevale F, Romano I, Romano V. Le mallatie dei lavoratori. Traducción al italiano del DE M ORBISARTIFICUM DIATRIBA, de Ramazzini B. Roma:N ueva Italia Científica, 1982:14.

2. León HS, Lara PE, Camarena 0 J. Historia y filosofía de la medicina. México, D.F.: Instituto Politécnico N acional, 2000: 155.

3. Gramsci A. 0 bras. Cuadernos de la cárcel: El risogimento. México, D.F.: Ed. Juan Pablos, 1980: 30-31.
4. Koyré A. Del mundo cerrado al universo infinito. 8a edición. México, D.F.: Siglo XXI Editores, 1992: 1.

5.A raujo JM. Las enfermedades de los trabajadores. Traducción al español del DE MORBIS ARTIFICUM DIATRIBA, de Ramazzini B. México, D. F.: Profedet, Miguel Angel Porrúa y UAM-X, 2000: 55-56.

6. Carnevale F, Romano I, Romano V. Le mallatie dei lavoratori.Traducción al italiano del DE M ORBISARTIFICUM DIATRIBA, de Ramazzini B. Roma:N ueva Italia C ientífica, 1982:78.

7. Masi E. La vida y la obra de Bernardino Ramazzini; Actas V Congreso $N$ acional Médico del Trabajo; 1922 junio 11-14; Florencia, Italia. En: Carnevale F, Romano I, Romano V. Le mallatie dei lavoratori.Traducción al italiano del DE M ORBISARTIFICUM DIATRIBA, de Ramazzini B. Roma:N ueva Italia C ientífica, 1982:16.

8. D evoto L. En el 200 aniversario de la muerte de Bernardino Ramazzini. II Lavoro 1914;7:305-313. En C arnevale F, Romano I, Romano V. Le mallatie dei lavoratori.Traducción al italiano del DE MORBIS ARTIFICUM DIATRIBA, de Ramazzini B. Roma: N ueva Italia C ientífica, 1982:17.

9. Piccinini P, Ramazzini B, Morgagni G B. En relación con la medicina del trabajo. Riv Storia sc Med e Natur 1930;21:264-271. En: Carnevale $F_{\text {, }}$ Romano I, Romano V. Le mallatie dei lavoratori.Traducción al italiano del DE MORBISARTIFICUM DIATRIBA, de Ramazzini B. Roma: $\mathrm{N}$ ueva Italia Científica, 1982:17.

10. Panseri G. El nacimiento de la policía médica: la organización sanitaria en los diferentes estados italianos. Historia de Italia,A nales 3.Turín: Einaudi, 1980:187-190. En:Carnevale F, Romano I, Romano V. Le mallatie dei lavoratori. Traducción al italiano del DE M ORBIS ARTIFICUM DIATRIBA, de Ramazzini B. Roma: N ueva Italia Científica, 1982:20.

11. Conti L. Perfil de Bernardino Ramazzini. Med Intern 1941; 49:79-78. En: Carnevale F, Romano I, Romano V. Le mallatie dei lavoratori.Traducción al italiano del DE MORBIS ARTIFICUM DIATRIBA, de Ramazzini B. Roma: N ueva Italia Científica, 1982:20.

12. Prenuda L.Trabajo y enfermedad: los fundamentos iluminísticos de la cuestión. En:Trabajo y enfermedad, perspectiva histórica y actual. Padua: Cedam, 1975:9-25. En: Carnevale F, Romano I, Romano V. Le mallatie dei lavoratori.Traducción al italiano del DE MORBIS ARTIFICUM DIATRIBA, de Ramazzini B. Roma: N ueva Italia Científica, 1982:20.

13. Braverman D. Muerte en el trabajo. México, D.F.: Editorial Siglo XXI Editores, 1983:9.

14. Kumate J. La edición de México en el Tricentenario. En:Araujo JM. Las enfermedades de los trabajadores. Traducción al español del DE M ORBIS ARTIFICUM DIATRIBA, de Ramazzini B. México, D. F:: Profedet, Miguel Angel Porrúa y UAM-X, 2000:8-9.

15. Entralgo LP. H istoria de la medicina. Barcelona: Ed. Salvat, 1982:251. 16. Menéndez EL. El modelo médico y la salud de los trabajadores. En: Basaglia F, Giovannini E, Maniati S, Pintor L, Pinella A et al. La salud de los trabajadores. 3å edición. México, D.F.: Editorial N ueva Imagen, 1981:11-51. 17. Fernández $0 \mathrm{~J}$. La lucha de los trabajadores por su salud. México, D.F.: Ediciones de la Universidad O brera de México, 1999:32. 